\title{
BMJ Open Metabolic Surgery Supporting Aftercare via Group-Intervention (MeSSAGES): study protocol of a randomised controlled trial
}

\author{
Eva-Maria Skoda, ${ }^{1}$ Jasmin Steinbach (D) , ${ }^{1}$ Anita Robitzsch, ${ }^{1}$ Corinna Pfeiffer, ${ }^{1}$ \\ Lynik Schüren, ${ }^{2}$ Jörg Tomaszewski, ${ }^{3}$ Marco Niedergethman, ${ }^{2}$ Adam Schweda, ${ }^{1}$ \\ Alexander Bäuerle (D) , ${ }^{1}$ Martin Teufel (D) ${ }^{1}$
}

To cite: Skoda E-M, Steinbach J, Robitzsch A, et al. Metabolic Surgery Supporting Aftercare via GroupIntervention (MeSSAGES): study protocol of a randomised controlled trial. BMJ Open 2021;11:e053839. doi:10.1136/ bmjopen-2021-053839

- Prepublication history and additional supplemental material for this paper are available online. To view these files, please visit the journal online (http://dx.doi.org/10.1136/ bmjopen-2021-053839).

Received 25 May 2021 Accepted 24 November 2021

Check for updates

(c) Author(s) (or their employer(s)) 2021. Re-use permitted under CC BY-NC. No commercial re-use. See rights and permissions. Published by BMJ.

${ }^{1}$ Clinic for Psychosomatic Medicine and Psychotherapy, LVR Hospital Essen, University of Duisburg-Essen, Essen, Germany

${ }^{2}$ Department of Surgery, Alfried Krupp Klinikum Essen, Essen, Germany

${ }^{3}$ Johnson \& Johnson Medical GmbH, Norderstedt, Germany

Correspondence to

Dr Eva-Maria Skoda

eva-maria.skoda@uni-due.de

\section{ABSTRACT}

Introduction Obesity is a constantly rising and costintensive medical issue worldwide. Severe obesity often needs surgery to promote weight loss, but due to the rapid therapeutic success after the surgery, many patients lack the awareness of the need to consistently maintain the postoperative care. However, therapeutic success and psychological well-being can be increased through group interventions and social support of the group members. Therefore, aftercare via group intervention is a promising approach. In this prospective randomised controlled study, the self-efficacy in a social media-based interactive, psychoeducational intervention is to be tested.

Methods and analysis The intervention group will complete a social media-supported group intervention for 6 weeks with weekly postings of educative contents and the possibility to exchange in groups via anonymous avatars. The control group will receive treatment as usual (TAU) after the obesity surgery as recommended in the German S3-guidelines Obesity Surgery and Metabolic Surgery. We will examine the effectiveness of a social media-supported intervention group, and therefore, the change in self-efficacy expectation. For the primary outcome, we will perform a mixed analysis of variance with time as the within-subject factor (times of measurement T0-T4) and the group assignment as the between-subject factor (intervention + TAU vs TAU group). Ethics and dissemination The study was approved by the Medical Association North Rhine (Ärztekammer Nordrhein, 2020031) and the patient enrolment will begin in July 2021.

Trial registration number DRKS00018089.

\section{INTRODUCTION}

\section{Background and rationales}

Obesity describes an above-average increase in body fat and is based on an imbalance between energy intake and consumption. ${ }^{1}$ Obesity is associated with a number of somatic comorbidities such as diabetes, musculoskeletal and cardiovascular diseases as well as carcinomas. $^{2}$ As a result, the life expectancy of people with obesity is considerably lower. ${ }^{34}$
Strengths and limitations of this study

- The study is an innovative approach to assess an online-based group intervention.

- Cost-effective and low threshold.

- Much of the success depends on the motivation and self-initiative of the group.

On a psychological level, the dysregulation of the psychoimmuno-neuroendocrine network as well as dissatisfaction with one's own body image, feelings of insufficiency and the resulting distress can result in development of depressive symptoms. ${ }^{5}$ Prior studies could show that people with a previous depressive illness suffer from obesity more often, but also that people with obesity have an increased risk of developing major depression and other diseases from the affective spectrum. ${ }^{6}$ Overall, people with obesity have an increased lifetime prevalence of mental illnesses. $^{7}$ Additionally, it was shown that people with an increased body mass index (BMI) often report a lower quality of life than people of normal weight. ${ }^{89}$

In the last decades, the worldwide prevalence of obesity has risen constantly. ${ }^{1}$ In 2017, the resulting medical costs of obesity in Germany amounted to over 13 billion euros. ${ }^{10}{ }^{11}$ Established treatment options are nutritional, behavioural as well as exercise therapies with the aim of not only reducing weight but also producing long-term maintenance of weight loss. ${ }^{12}$ In case of severe obesity, surgery is the only effective therapy in most cases, which not only leads to initially verifiable weight reduction, but also to longterm success. $^{13-16}$ In 2014, 9.225 obesity surgeries were conducted in Germany. ${ }^{17}$ Even though obesity surgery patients benefit with 
respect to weight loss and decrease in weight-related somatic disorders as well as improvements in depressive symptoms, there are still some patients at-risk for a new onset of depression and suicide. ${ }^{18}$ Therefore, the inclusion of mental health professionals is integral to help developing and evaluating interventions with respect to possibly occurring depression symptoms, suicidal ideation and other mental health disorders after obesity surgery. Consolidating resources is particularly important with regard to therapy options for patients. There is evidence that psychological resources are associated with a lower incidence of depressive symptoms. ${ }^{19}$

However, the long-term outcomes largely depend on patient adherence. ${ }^{20}$ Due to the rapid onset of measurable success after an obesity surgery, many patients lack awareness of the need to consistently maintain the recommended postoperative care. Therefore, it is important to promote compliance through innovative types of aftercare, such as group programmes, video conferences or media-based possibilities such as smartphone apps in order to allow for low-threshold access. ${ }^{20}{ }^{21}$ It has been shown that attending group meetings in which experiences can be shared and thus the experience of social support is delivered, resulting in a statistically positive effect on weight loss. ${ }^{22-24}$

In the 'BaSe' study, a follow-up via videoconference has caused no weight loss or increase in quality of life, but a reduction in depressive symptoms and an increase in self-efficacy. ${ }^{21}{ }^{25}$ Internet-based aftercare programmes show advantages such as flexibility in terms of time, location and broad access, ${ }^{26}$ which means that patients who struggle with motivation or who live too far away can participate in an intervention. ${ }^{27}$ Social networks are applications that not only enable users to formulate and pass on content themselves, but also facilitate to benefit from contributions from other users. ${ }^{28} \mathrm{~A}$ review of recent research about social media and obesity in adults showed that social media has the potential to connect these people with social support for weight loss and that it is used not only widely but also in different kinds of ways with varying patient engagement. ${ }^{29}$ Greater involvement of participants in the social media component predicts better outcome in weight loss. ${ }^{30-32}$ For example, in an intervention that only took place in a private Facebook group, the number of comments and likes correlated strongly with the proportional weight loss of the participants after 12 weeks. ${ }^{33}$ Regular provision of content that actively encourages commitment is more effective than waiting for spontaneous, unguided content. Greater number of posts reporting a healthy act, asking for help, making a plan, reporting weight or confirming other participants were associated with greater weight loss. ${ }^{33}$ In a study in which overweight students were provided with evidence-based content on the topic of weight loss via a Facebook group, the social network proved to be a suitable and easy-to-use medium. ${ }^{34}$ Facebook seems to be an adequate means to offer aftercare and health information, especially in the case of obesity. With one of the main functions of Facebook, namely communicating with one another, ${ }^{35}$ it even seems ideal to reinforce a desired social support in a group setting. This is supported by the fact that Facebook already creates an environment in which users like to share information themselves. ${ }^{36}$

In addition, social networks offer the opportunity to minimise the aforementioned medical costs. ${ }^{37}$ Many patients can be reached at the same time through social networks ${ }^{37}$ without taking up the time of physicians or therapists. $^{38}$

Successful weight loss and psychological well-being can, therefore, be promoted through group interventions and social support, which is why further research is a promising approach. However, existing offers are cost-intensive and involve much of effort.

The current study situation suggests that follow-up care after an obesity surgery is necessary to guarantee long-term success. An increasing demand due to the mounting number of surgical interventions and the need for a structured aftercare, not only surgical and dietetic but also psychotherapeutic, represents an economic challenge that can be met in group programmes. Group programmes via social networks seem to represent a lowthreshold and cost-effective perspective, which facilitate therapy adherence. Despite some promising approaches, so far there is no clear empirical evidence on the topic.

A randomised controlled study with the purpose of supporting patients after obesity surgery is urgently required. Our aim is to close this gap.

\section{Objectives and trial design}

The aim of the study is to offer group-based follow-up care for patients after obesity surgery using a randomised controlled design. We expect the MeSSAGES intervention to be superior to treatment as usual (TAU) in terms of self-efficacy (primary outcomes), as well as differences in the dependent variables like weight loss (BMI), change of eating behaviour, depression symptoms, quality of life, subjective level of stress and general health status (secondary outcomes). Furthermore, acceptance and satisfaction with the intervention will be evaluated (tertiary outcomes).

\section{METHODS: PARTICIPANTS, INTERVENTIONS AND OUTCOMES}

In general, this study protocol will be reported in accordance with the Standard Protocol Items: Recommendations for Interventional Trials guidelines for randomised controlled studies (see online supplemental table 1).

\section{Patient and public involvement}

Patients or the public were not involved in the design, or conduct, or reporting, or dissemination plans of our research.

\section{Study setting}

Our prospective randomised controlled study is designed to examine the efficacy of a psychoeducative group intervention via Facebook after an obesity surgery. The trial 


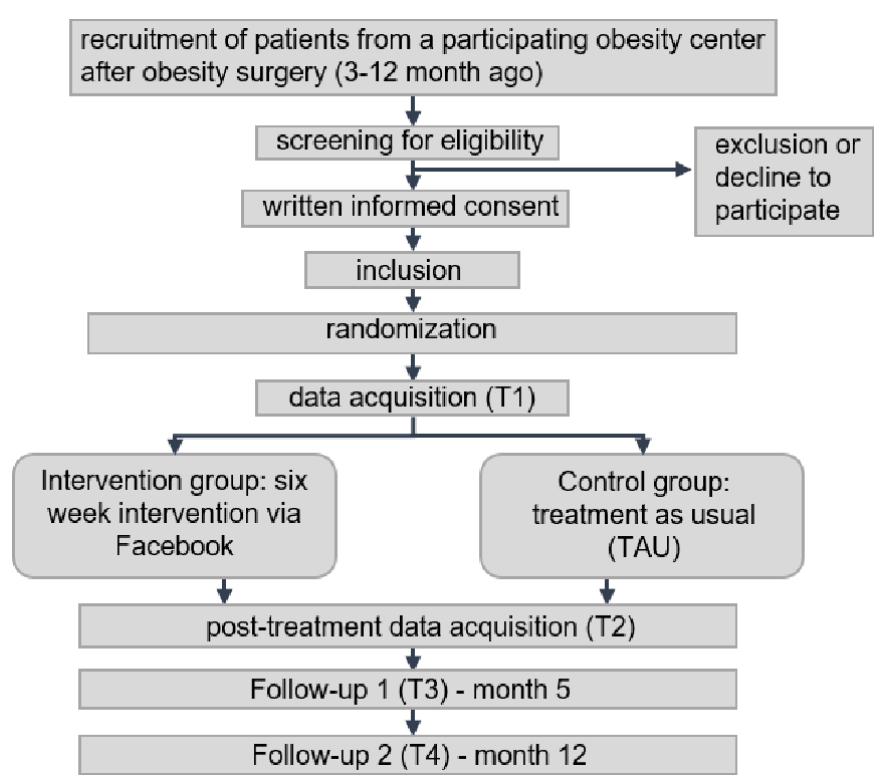

Figure 1 Participant timeline. Diagram of the study schedule with measure times T1 (preintervention), T2 (after intervention), T3 (5 months follow-up) and T4 (12-month follow-up).

flow is shown in figure 1. The intervention group (IG) will complete a Facebook-supported group intervention for 6 weeks with weekly postings of educative contents and the possibility to exchange in groups via anonymous avatars. The control group (CG) will receive regular aftercare after the surgery as recommended in the German S3-Guidelines Obesity Surgery and Metabolic Surgery (40a). Additionally, psychometric data will be collected before (T1) and after treatment (T2) as well as in a follow-up of 5 months (T3) and twelve months (T4) after the intervention.

\section{Participant eligibility}

We applied a number of eligibility criteria. Participants will be included provided that they are at least 18 years of age, had an obesity surgery due to morbid obesity (BMI $>40 \mathrm{~kg} / \mathrm{m}^{2}$ or BMI $>35 \mathrm{~kg} / \mathrm{m}^{2}$ with comorbidities), the surgery was 3-12 months ago, and have given their informed consent. Patients with no internet access, no or insufficient internet knowledge, unstable psychopathological conditions or insufficient knowledge of German language will be excluded.

\section{Intervention}

CG (TAU)

The study participants will be randomly assigned to the two groups-the IG and the CG (see figure 1). The CG will receive TAU, which is based on the German S3 Guidelines Obesity Surgery and Metabolic Surgery. ${ }^{12}$ The guidelines represent the state of the art treatment for obesity surgery and metabolic surgery in Germany. This includes multidisciplinary evaluation and preparation, conservative and surgical treatment elements, and a lifelong follow-up.
Metabolic surgery supporting aftercare via group intervention For the IG participants, pseudonymised avatar profiles will be set up on Facebook, which can be accessed via a 1 min email address. We will split the intervention group in smaller groups of 8-10 because of the different start times of patients after surgery. The avatars in the intervention will only take part in a closed Facebook group of 8-10 patients and exchange ideas with the other intervention participants in the group. An exchange with nonstudy participants on Facebook is not permitted and a confidentiality declaration must be filled out in advance. During the 6-week online intervention, the subjects are given an intervention unit in the Facebook group every week. Contents are psychoeducational information texts, graphs and flow charts to gain knowledge about coping strategies with binge eating, stress management, social interaction, exercise behaviour, self-worth and relapse prevention. They are intended to encourage patients to deal more intensively with their situation self-guided (without therapeutic guidance) and to share ideas with other affected persons about the topics and possible problems. Additionally, the participants are encouraged to take part in the discussions.

The topics of the intervention units are structured in (1) postoperative eating behaviour and binge eating, (2) stress management and eating behaviour, (3) social interaction and dealing with conflicts, (4) exercise behaviour/ motivating aspects, (5) self-worth and (6) outlook and relapse prevention (see table 1 ).

\section{Materials and instruments}

The study is based on the social network and messengerplatform Facebook, where users can communicate with each other, post comments, share photographs or other contents, and group pages can be created for social exchange. For this study, we will create a closed and private group, which will be deleted at the time of the study completion. Additionally, accounts and avatars will be built for the participants and will also be deleted after the intervention. The avatars are anonymised with flower names and pictures for females and tree names and pictures for males. The study nurse's and psychologists' names are also anonymised.

\section{Outcomes}

The administration of intervention materials, access keys for accounts, introduction of group rules, and, if necessary, dialogue moderation is performed by study staff, consisting of a study nurse and a M.Sc. Psychologist. Height and weight are measured at times T1 -T4. The patients themselves convey their data via email or telephone. In addition, the patients fill out standardised psychometric online questionnaires.

These questionnaires contain basic documentation, self-efficacy, social support, eating behaviour, subjective level of stress, depression and evaluation and rating of the intervention, as shown in table 2. 
Table 1 Modules of the intervention with topic, psychoeducation and skills

\begin{tabular}{|c|c|c|c|}
\hline Module & Topic & Psychoeducation & Skills \\
\hline $\begin{array}{l}\text { Coping with } \\
\text { binge eating }\end{array}$ & $\begin{array}{l}\text { Postoperative eating } \\
\text { behaviour and binge } \\
\text { eating }\end{array}$ & $\begin{array}{l}\text { Rapid and significant weight loss after obesity surgery can } \\
\text { lead to negligence with regard to eating behaviour. It is } \\
\text { therefore important to prevent uncontrolled eating early and } \\
\text { consistently. }\end{array}$ & $\begin{array}{l}\text { Risk factors } \\
\text { Motivation } \\
\text { Relapse prevention }\end{array}$ \\
\hline $\begin{array}{l}\text { Stress } \\
\text { management }\end{array}$ & $\begin{array}{l}\text { Stress management } \\
\text { and eating behaviour }\end{array}$ & $\begin{array}{l}\text { Especially after a gastric resection, it is important to stay } \\
\text { tuned and address factors that affect your weight loss and } \\
\text { general well-being. }\end{array}$ & $\begin{array}{l}\text { Coping with stress } \\
\text { factors } \\
\text { Alternative behaviour }\end{array}$ \\
\hline $\begin{array}{l}\text { Exercise } \\
\text { behaviour }\end{array}$ & $\begin{array}{l}\text { Exercise behaviour/ } \\
\text { motivating aspects }\end{array}$ & $\begin{array}{l}\text { Even after a gastric-reducing operation, physical activity is } \\
\text { essential to achieve and maintain a healthy body weight. }\end{array}$ & $\begin{array}{l}\text { Concrete and realistic } \\
\text { goals } \\
\text { Motivation }\end{array}$ \\
\hline Self-worth & Increase self-worth & $\begin{array}{l}\text { In order to strengthen self-esteem, it is important to discover } \\
\text { and appreciate positive traits in yourself. }\end{array}$ & $\begin{array}{l}>\text { Self-efficacy } \\
>\text { Self-worth }\end{array}$ \\
\hline
\end{tabular}

The Psychotherapy Basic Documentation (Psy-BADO) is a standardised psychosomatic-psychotherapeutic basis documentation used in the Clinic for Psychosomatic Medicine and Psychotherapy. ${ }^{39}$ The Psy-BADO includes basic questions about demographics and therapy goals.

The general expectation of Self-Efficacy Expectation Scale (SWE) is measured with ten items on a four-point Likertscale $\left(0=\right.$ 'strongly disagree' to $3=$ 'strongly agree'). ${ }^{40} \mathrm{SWE}$ measures the optimistic expectation of competence, that is, the confidence in mastering a difficult situation.

The 12-Item Short-Form Survey (SF-12) is a selfreported measure assessing quality of life. ${ }^{41}$
The Eating Disorder Examination-Questionnaire (EDE-Q) is a 28-items questionnaire. ${ }^{42}$ The EDE-Q is used to assess the specific psychopathology of eating disorders in adults and adolescents with the subscales Restraint, Eating Concern, Weight Concern and Shape Concern.

The Personal Health Questionnaire Depression Scale-8 (PHQ-8) measures depression symptoms with eight items from $0=$ 'not at all' to $3=$ 'nearly every day'. 43

The Perceived Stress Questionnaire (PSQ) assesses general subjective burden with 30 items and seven subscales (harassment, overload, irritability, lack of joy, fatigue, worries, tension) from $0=$ 'almost never' to 3 = 'usually' ${ }^{44}$

Table 2 Questionnaires and times of measurement

\begin{tabular}{|c|c|c|c|c|}
\hline Measures & $\begin{array}{l}\text { T1: Postsurgery/ } \\
\text { preintervention }\end{array}$ & $\begin{array}{l}\text { T2: after } \\
\text { intervention }\end{array}$ & $\begin{array}{l}\text { T3: follow-up } \\
5 \text { months }\end{array}$ & $\begin{array}{l}\text { T4: follow-up } \\
12 \text { months }\end{array}$ \\
\hline \multicolumn{5}{|l|}{ Primary outcome } \\
\hline \multicolumn{5}{|c|}{ Secondary outcome } \\
\hline $\mathrm{BMI}$ & $x$ & $x$ & $x$ & $x$ \\
\hline PHQ-8 & $x$ & $x$ & $x$ & $x$ \\
\hline $\mathrm{PSQ}$ & $x$ & $x$ & $x$ & $x$ \\
\hline SF-12 & $x$ & $x$ & $x$ & $x$ \\
\hline FSozU & $x$ & $x$ & $x$ & $x$ \\
\hline $\begin{array}{l}\text { Evaluation of } \\
\text { intervention* } \\
\text { - Acceptance } \\
\text { - Satisfaction }\end{array}$ & & $X$ & & \\
\hline
\end{tabular}

*Self-Generated Questionnaire.

BMI, body mass index; EDE-Q, Eating Disorder Examination-Questionnaire; FSozU, Social Support Questionnaire; PHQ-8, Personal Health Questionnaire Depression Scale-8; PSQ, Perceived Stress Questionnaire; SF-12, 12-Item Short-Form Survey; SWE, Self-Efficacy Expectation Scale. 
The Social Support Questionnaire (FSozU) is a SF-22 ${ }^{45}$ and measures the subjective perceived social support in the social environment.

Additionally, we will use a self-generated questionnaire for evaluation of the intervention containing 43 items about the intervention modules, Facebook as an intervention platform and social exchange. The questionnaire contains open questions as well as different types of Likert scales.

\section{Primary outcome measures}

As the primary outcome, we will examine the perceived self-efficacy of a Facebook-supported group intervention in patients who underwent obesity surgery. Therefore, the change in self-efficacy expectation is measured via the SWE with 10 items at four dates (T1 before treatment, T2 directly after treatment, T3 5 months after the intervention and T4 12 months after treatment). Our primary end point will be T2 directly after the treatment. The differences between the times of measurement will be expressed as score differences.

\section{Secondary outcome measures}

As the secondary outcome, we monitor additional weight loss, change in eating behaviour, depression, quality of life, subjective level of stress and general health status measured via weight measures, EDE-Q with 28 items, PHQ-8 with eight items, the PSQ with five items, a SF of the general health status (SF-12) and a FSozU with 22 items. With the 43-item self-created questionnaire, the intervention is evaluated considering acceptance and satisfaction.

\section{Trial timeline}

We assume that the total time for patient enrolment will take approximately 2 years. The online intervention is planned for 6 weeks. Before and after, data is assessed using standardised questionnaires (T1 and T2). A third survey takes place after 5 months and 12 months (T3, T4). Thus, for the individual, the duration of the whole trial is about 12 months, of which only 6 weeks are actively spent in the intervention. For further information, see figure 1.

\section{Sample size calculation}

The goal is to recruit 80 patients (40 IG, 40 CG) through the follow-up consultation of the obesity surgery centre in Essen. We calculated the sample size by considering a correlation as a reliable approximation of the relationship between our measurement times. Jerusalem and Schwarzer ${ }^{40}$ give a 1-year inter-rater reliability of $\mathrm{r}=0.54$ for the questionnaire on general expectation of SWE, so that we consider a correlation of $\mathrm{r}=0.50$ as a reliable approximation of the relationship between our measurement times. With this value, 80 test participants, an a-error of $\alpha=0.05$, an effect with an effect size of $\mathrm{f}=0.155$ and larger can be determined at a power of $1-\beta=0.95$, which, according to Cohen, is a small to medium effect size (Cohen, 1992). Small to medium effect sizes were also found for other e-health studies on psychosocial interventions (Massoudi $e t a l$, Lamb et al), so we assume that our design can reliably determine group differences over time. Even in the case of a high drop-out rate of over $35 \%$, we would be able to discover similar effect sizes with a power of $1-\beta=0.80$ (with exactly 52 participants). However, we overall expect a low drop-out rate of no more than $10 \%$, similar to other intervention studies (eg, Iacovino et al).

\section{Recruitment}

Patients will be recruited through the follow-up consultation of a German obesity surgery centrum by the local coordinator. Before participation, informed consent must be given.

\section{METHODS: ASSIGNMENT OF INTERVENTIONS Allocation and blinding}

The patients are allocated to either the IG or the CG by using a random number generator. They will be informed by the clinicians or researcher who were, therefore, not blinded. There was no attempt to blind clinicians or researchers since our study is an intervention study where the clinicians and researchers managed the Facebook groups and intervention materials. However, there is no expected influence regarding to the outcome since the outcome is measured with mostly validated questionnaires and the biometrician is blinded.

\section{METHODS: DATA COLLECTION, MANAGEMENT AND ANALYSIS Data collection methods and management}

The personal and psychological data of the online questionnaires, which is collected in connection with this study (eg, personal or psychological data and evaluations of the questionnaires), will be stored on a protected hard drive of the study director at the clinic for the duration of the data collection and evaluation. The data will be available in pseudonymised form and a code will be assigned to each patient. If necessary, a return can be made using a list of subjects. This list will be kept separately from the data, locked and only accessible to the study management. The data will be archived and stored for 10 years after trial completion and will be deleted after this time. The possibility for patients to revoke their participation and have their data deleted is set out in the data protection declaration.

Facebook as an external online social network has its own data protection guidelines that the study directors cannot influence. This poses the necessity of detailed education of the study participants about the data protection guidelines of Facebook and the obtaining of informed consent from the study participants. Participants will not use their own Facebook profiles to visit the Facebook group, but will receive pseudonymised avatars from us that do not allow any conclusions to be drawn about their own profiles. These avatars are set up with $1 \mathrm{~min}$ emails and a confidentiality declaration by the test subjects is drawn up with special reference to communication with other Facebook users with an anonymised avatar. 


\section{Statistical methods}

Before analysis, standard tests of normality and homogeneity of variance and additional descriptive analyses of the sociodemographic data will be performed. The variables age and sex will be used to impute missing values with SPSS (IBM: V.26). For the primary analysis of the data a $2 \times 5$ mixed repeated measures analysis of variance with time as the innersubject factor (measurement times t0 to 4 ) and the group assignment as the between-subject factor (intervention +TAUvs TAU group) will be used. For the primary outcome, change of SWE over time between IG and CG will be compared. For the secondary outcome, change of BMI, EDE-Q, PHQ-8, PSQ, SF-12 and FSozU over time between the two groups will be examined. Additionally, the evaluation of the intervention will be put in relation to our results.

\section{METHODS: MONITORING \\ Data monitoring}

The data monitoring will be established by the clinics study staff and the local ethics committee.

\section{Harms and auditing}

The validated and self-generated questionnaires do not deliver specific health risks or side effects concerning patient safety. Thus, auditing is not intended. In the case of psychiatric crisis (suicidality) or somatic crisis, the study will be terminated for the subject and inpatient or stationary treatment will be initiated. It is possible that the comment function may lead to differences between the opinions of participants of the IG, which will be moderated by the study staff at all times.

\section{ETHICS AND DISSEMINATION \\ Research ethics}

The study was approved by the Medical Association North Rhine (Ärztekammer Nordrhein, 2020031) and the intervention will begin in summer, 2021.

\section{Protocol amendments}

Important protocol modification will be communicated to the Medical Association North Rhine.

\section{Consent or assent}

The study staff (coordinator of the obesity surgery centre) will contact potential participants and obtain written informed consent.

\section{Confidentiality}

To protect confidentiality, the participant's data will be pseudonymised and stored for at least 10 years. The pseudonymised data will be in a locked file.

\section{Declaration of interest}

The authors declare that they have no financial or competing interest.

\section{Access to data}

The database is only accessible for the study staff and researchers of the project.

Ancillary and post-trial care

Not applicable.

\section{Dissemination policy}

Results will be disseminated in peer-reviewed journals as well as conference presentations.

\section{DISCUSSION}

Obesity is a constantly increasing and cost-intensive medical issue worldwide because of its long-time somatic consequences, increased lifetime prevalence of mental illnesses and comorbidities in general $(1-10,15,16)$. Obesity surgery is the most effective treatment and hence important to reduce the overall medical issues and psychological health problems in long term. Accordingly, retained effects depend on psychological factors, especially compliance (21). Compliance and self-efficacy can be enhanced by (social network) group meetings and its social support. Facebook is a frequently used social platform, which is cost-effective as well as low-threshold and can be used as an easy-to-use aftercare for patients after an obesity surgery. The feasibility of this kind of intervention shall be tested in the present intervention study.

The MeSSAGES study seeks to assess the effectiveness of a low-threshold and cost-effective psychological follow-up care after obesity surgery with Facebook as a platform. Healthcare system should respond to these needs of a low-threshold, cost-effective intervention. Since only few data exists to this point of time, our study has relevance. The findings on the applicability and manageability of this medium as an aftercare intervention are made possible with the MeSSAGES study. A previous study indicated that a follow-up after-treatment-group via videoconference leads to a reduction in depressive symptoms, but no differences in weight loss. ${ }^{21}$ We expect MeSSAGES to prove the effectiveness of the Facebook-supported group intervention with a positive change in self-efficacy expectation and additionally, weight loss, a change in eating behaviour, depression, quality of life, subjective level of stress and general health status.

One limitation of the study is that much of the intervention's success depends on the motivation and self-initiative of the group participants. Furthermore the researchers (except for the biometrician) are not blinded. However, we do not expect any influence regarding the outcome since the patients are pseudomyzed in the Facebook groups, but in future studies an examining approach could be integrated. Further studies could also expand the approach and examine the effectiveness of group interventions in other social media platforms. Overall, the MeSSAGES study is an innovative, novel approach and the outcome is yet to be evaluated. 
Contributors E-MS designed the study initially, developed the intervention and administered the trial. She helped with the manuscript as well as accepts full responsibility for the finished work and controlled the decision to publish. JS contributed to designing the study. She provided the draft and adapted it. She is trial site investigators who contributed to refinement of the study design and protocol, implementation of the trial and who are involved regarding patient recruitment, treatment and data collection. AR contributed to designing the study and helped with the manuscript. She is trial site investigators who contributed to refinement of the study design and protocol, implementation of the trial and who are involved regarding patient recruitment, treatment, and data collection. CP contributed to designing the study and helped with the manuscript. She is trial site investigators who contributed to refinement of the study design and protocol, implementation of the trial and who are involved regarding patient recruitment, treatment and data collection. LS contributed to designing the study and helped with the manuscript. She is trial site investigator who contributed to refinement of the study design and protocol, implementation of the trial and who are involved regarding patient recruitment, treatment, and data collection. JT contributed to designing the study and helped with the manuscript. He is trial site investigator who contributed to refinement of the study design and protocol, implementation of the trial and who are involved regarding patient recruitment, treatment, and data collection. MN contributed to designing the study and helped with the manuscripts. He is trial site investigator who contributed to refinement of the study design and protocol, implementation of the trial and who are involved regarding patient recruitment, treatment, and data collection. AS provided statistical and methodical expertise in trial design and is the principal trial statistician. AS is responsible for data management during the trial. AB and MT are shared last author. They initiated the study and contributed to designing the study. They further adapted the intervention and the manuscript draft. All authors revised the manuscript critically and approved the final version to be published. All authors agree to be accountable for all aspects of the work in ensuring that questions related to the accuracy or integrity of any part of the work are appropriately investigated and resolved. $A B$ and MT are joint last authors.

Funding We acknowledge support by the Open Access Publication Fund of the University of Duisburg-Essen.

Competing interests None declared.

Patient consent for publication Not applicable.

Provenance and peer review Not commissioned; externally peer reviewed.

Supplemental material This content has been supplied by the author(s). It has not been vetted by BMJ Publishing Group Limited (BMJ) and may not have been peer-reviewed. Any opinions or recommendations discussed are solely those of the author(s) and are not endorsed by BMJ. BMJ disclaims all liability and responsibility arising from any reliance placed on the content. Where the content includes any translated material, BMJ does not warrant the accuracy and reliability of the translations (including but not limited to local regulations, clinical guidelines, terminology, drug names and drug dosages), and is not responsible for any error and/or omissions arising from translation and adaptation or otherwise.

Open access This is an open access article distributed in accordance with the Creative Commons Attribution Non Commercial (CC BY-NC 4.0) license, which permits others to distribute, remix, adapt, build upon this work non-commercially, and license their derivative works on different terms, provided the original work is properly cited, appropriate credit is given, any changes made indicated, and the use is non-commercial. See: http://creativecommons.org/licenses/by-nc/4.0/.

\section{ORCID iDs}

Jasmin Steinbach http://orcid.org/0000-0002-2738-4089

Alexander Bäuerle http://orcid.org/0000-0003-1488-8592

Martin Teufel http://orcid.org/0000-0003-2120-1840

\section{REFERENCES}

1 Ogden CL, Yanovski SZ, Carroll MD, et al. The epidemiology of obesity. Gastroenterology 2007;132:2087-102.

2 WHO. Obesity and overweight, 2020. Available: https://www.who.int/ news-room/fact-sheets/detail/obesity-and-overweight

3 Mensink GBM, Schienkiewitz A, Haftenberger M, et al. [Overweight and obesity in Germany: results of the German Health Interview and Examination Survey for Adults (DEGS1)]. Bundesgesundheitsblatt Gesundheitsforschung Gesundheitsschutz 2013;56:786-94.

4 Carlsson LMS, Sjöholm K, Jacobson P, et al. Life expectancy after bariatric surgery in the Swedish obese subjects study. $N$ Engl $\mathrm{J}$ Med 2020;383:1535-43.
5 Patist CM, Stapelberg NJC, Du Toit EF, et al. The brain-adipocytegut network: linking obesity and depression subtypes. Cogn Affect Behav Neurosci 2018;18:1121-44.

6 Gadalla TM. Association of obesity with mood and anxiety disorders in the adult general population. Chronic Dis Can 2009;30:28-35.

7 Simon GE, Von Korff M, Saunders K, et al. Association between obesity and psychiatric disorders in the US adult population. Arch Gen Psychiatry 2006;63:824-30.

8 Taylor VH, Forhan M, Vigod SN, et al. The impact of obesity on quality of life. Best Pract Res Clin Endocrinol Metab 2013;27:139-46.

9 Avila C, Holloway AC, Hahn MK, et al. An overview of links between obesity and mental health. Curr Obes Rep 2015;4:303-10.

10 Konnopka A, Dobroschke A, Lehnert T, et al. [The Costs of Overweight and Obesity: a Systematic Review]. Gesundheitswesen 2018;80:471-81.

11 Knoll KP, Hauner H. Kosten Der Adipositas in Der Bundesrepublik Deutschland. Adipositas - Ursachen, Folgeerkrankungen, Therapie 2017;2:204-10.

12 Teufel M, Stefan N, Kunze D. Interdisziplinäre Leitlinie der Qualität S3 zur "Prävention und Therapie der Adipositas". Adipositas - Ursachen, Folgeerkrankungen, Therapie 2017;08:179-221.

13 Buchwald $\mathrm{H}$, Avidor $\mathrm{Y}$, Braunwald $\mathrm{E}$, et al. Bariatric surgery: a systematic review and meta-analysis. JAMA 2004;292:1724-37.

14 Sjöström L, Lindroos A-K, Peltonen M, et al. Lifestyle, diabetes, and cardiovascular risk factors 10 years after bariatric surgery. $N$ Engl J Med 2004;351:2683-93.

15 O'Brien PE, Hindle A, Brennan L, et al. Long-Term outcomes after bariatric surgery: a systematic review and meta-analysis of weight loss at 10 or more years for all bariatric procedures and a singlecentre review of 20-year outcomes after adjustable gastric banding. Obes Surg 2019;29:3-14.

16 Arterburn DE, Telem DA, Kushner RF, et al. Benefits and risks of bariatric surgery in adults: a review. JAMA 2020;324:879-87.

17 GEK-Report B. BARMER GEK report Hospital 2016, 2016. Available: https://www.barmer.de/presse/infothek/studien-und-reports/ krankenhausreport/report-2016-39066

18 Müller A, Hase C, Pommnitz M, et al. Depression and suicide after bariatric surgery. Curr Psychiatry Rep 2019;21:84.

19 Robitzsch A, Schweda A, Hetkamp M, et al. The impact of psychological resources on body mass index in obesity surgery candidates. Front Psychiatry 2020;11:649.

20 Hood MM, Corsica J, Bradley L, et al. Managing severe obesity: understanding and improving treatment adherence in bariatric surgery. J Behav Med 2016;39:1092-103.

21 Wild B, Hünnemeyer K, Sauer $\mathrm{H}$, et al. A 1-year videoconferencingbased psychoeducational group intervention following bariatric surgery: results of a randomized controlled study. Surg Obes Relat Dis 2015;11:1349-60.

22 Hildebrandt SE. Effects of participation in bariatric support group after Roux-en-Y gastric bypass. Obes Surg 1998;8:535-42.

23 Kaiser KA, Franks SF, Smith AB. Positive relationship between support group attendance and one-year postoperative weight loss in gastric banding patients. Surg Obes Relat Dis 2011;7:89-93.

24 Markert J, Herget S, Petroff D, et al. Telephone-based adiposity prevention for families with overweight children (T.A.F.F.-Study): one year outcome of a randomized, controlled trial. Int $J$ Environ Res Public Health 2014;11:10327-44.

25 Wild B, Hünnemeyer $\mathrm{K}$, Sauer $\mathrm{H}$, et al. Sustained effects of a psychoeducational group intervention following bariatric surgery: follow-up of the randomized controlled base study. Surg Obes Relat Dis 2017;13:1612-8.

26 Conceição EM, Machado PPP, Vaz AR, et al. APOLO-Bari, an Internet-based program for longitudinal support of bariatric surgery patients: study protocol for a randomized controlled trial. Trials 2016;17:114.

27 Fichter MM, Quadflieg N, NissImüller K, et al. Does Internet-based prevention reduce the risk of relapse for anorexia nervosa? Behav Res Ther 2012;50:180-90.

28 Vandelanotte C, Kirwan M, Rebar A, et al. Examining the use of evidence-based and social media supported tools in freely accessible physical activity intervention websites. Int J Behav Nutr Phys Act 2014;11:105.

29 Waring ME, Jake-Schoffman DE, Holovatska MM, et al. Social media and obesity in adults: a review of recent research and future directions. Curr Diab Rep 2018;18:34.

30 Pagoto SL, Waring ME, Schneider KL, et al. Twitter-Delivered behavioral weight-loss interventions: a pilot series. JMIR Res Protoc 2015;4:e123.

31 Turner-McGrievy GM, Tate DF. Weight loss social support in 140 characters or less: use of an online social network in a 
remotely delivered weight loss intervention. Trans/ Behav Med 2013;3:287-94.

32 Hales SB, Davidson C, Turner-McGrievy GM. Varying social media post types differentially impacts engagement in a behavioral weight loss intervention. Transl Behav Med 2014;4:355-62.

33 Pagoto S, Tulu B, Agu E, et al. Using the habit APP for weight loss problem solving: development and feasibility study. JMIR Mhealth Uhealth 2018;6:e145.

34 Napolitano MA, Hayes S, Bennett GG, et al. Using Facebook and text messaging to deliver a weight loss program to college students. Obesity 2013;21:25-31.

35 Buffardi LE, Campbell WK. Narcissism and social networking web sites. Pers Soc Psychol Bull 2008;34:1303-14.

36 Nadkarni A, Hofmann SG. Why do people use Facebook? Pers Individ Dif 2012;52:243-9.

37 Maher CA, Lewis LK, Ferrar K, et al. Are health behavior change interventions that use online social networks effective? A systematic review. J Med Internet Res 2014;16:e40.
38 Bradbury K, Dennison L, Little P, et al. Using mixed methods to develop and evaluate an online weight management intervention. $\mathrm{Br}$ $J$ Health Psychol 2015;20:45-55.

39 Heuft G, Senf W, Bell K, et al. Psy-BaDo. Psychotherapeut 1998;43:48-52.

40 Jerusalem MS, Schwarzer R. SWE - Skala zur Allgemeinen Selbstwirksamkeitserwartung. Trier: ZPID (Leibniz Institute for Psychology Information) - Testarchiv, 2003.

41 HSJ T-BD. Short form 12 health survey (SF-12). Springer: Dordrecht, 2014.

42 Berg KC. Eating Disorder Examination (EDE)/(EDE-Q). Singapore: Springer, 2016

43 Löwe B, Zipfel S, PHQ-D HW. Gesundheitsfragebogen für Patienten. Zeitschrift für Medizin 2003;12:90-3.

44 Shahid A, Wilkinson K, Marcu S. Perceived Stress Questionnaire (PSQ). In: Shahid A, Wilkinson K, Marcu S, eds. Stop, that and one hundred other sleep scales. New York, NY: Springer New York, 2012: 273-4.

45 Fydrich T, Sommer G, Brühler E. Fragebogen zur Sozialen Unterstützung (F-SozU) (survey of social support). Göttingen: Hogrefe, 2007. 